\title{
Bifurcation Analysis for Phage Lambda with Binding Energy Uncertainty
}

\author{
Ning Xu, ${ }^{1,2}$ Xue Lei, ${ }^{3}$ Ping Ao, ${ }^{3}$ and Jun Zhang ${ }^{1,2}$ \\ ${ }^{1}$ Joint Institute of UMich-SJTU, Shanghai Jiao Tong University, Shanghai 200240, China \\ ${ }^{2}$ Key Laboratory of System Control and Information Processing, Ministry of Education, Shanghai 200240, China \\ ${ }^{3}$ Shanghai Center for Systems Biomedicine, Shanghai Jiao Tong University, Shanghai 200240, China \\ Correspondence should be addressed to Jun Zhang; zhangjun12@sjtu.edu.cn
}

Received 30 October 2013; Accepted 25 December 2013; Published 3 February 2014

Academic Editor: Jose Nacher

Copyright (c) 2014 Ning Xu et al. This is an open access article distributed under the Creative Commons Attribution License, which permits unrestricted use, distribution, and reproduction in any medium, provided the original work is properly cited.

\begin{abstract}
In a phage $\lambda$ genetic switch model, bistable dynamical behavior can be destroyed due to the bifurcation caused by inappropriately chosen model parameters. Since the values of many parameters with biological significance often cannot be accurately acquired, it is thus of fundamental importance to analyze how and to which extent the system dynamics is influenced by model parameters, especially those parameters pertaining to binding energies. In this paper, we apply a Jacobian method to investigate the relation between bifurcation and parameter uncertainties on a phage $\lambda$ OR model. By introducing bistable range as a measure of system robustness, we find that RNA polymerase binding energies have the minimum bistable ranges among all the binding energies, which implies that the uncertainties on these parameters tend to demolish the bistability more easily. Moreover, parameters describing mutual prohibition between proteins Cro and CI have finite bistable ranges, whereas those describing self-prohibition have infinity bistable ranges. Hence, the former are more sensitive to parameter uncertainties than the latter. These results help to understand the influence of parameter uncertainties on the bifurcation and thus bistability.
\end{abstract}

\section{Introduction}

Bistability is a salient feature of phage $\lambda$ genetic switch. Mathematical models with a number of parameters have been established to describe its bistable behavior. However, parameter variation in phage $\lambda$ model may considerably change the system dynamics. Therefore, in the case when the model parameters are not precisely known, it is useful to study the influence of parameter uncertainty on system dynamics.

Since 1980s, researchers have developed several models for phage $\lambda$ genetic switch. Reference [1] proposed a quantitative model for phage $\lambda$ according to the principles of statistical thermodynamics. The model by [2] included two variables, that is, concentrations of proteins CI and Cro. Reference [3] extended this model and pointed out the discrepancy between experimental and theoretical results. Moreover, models with four variables were built to include more ingredients such as mRNA concentration in phage $\lambda$ [4]. Other useful models include Markov chain model [5], delayed reaction stochastic model [6], fuzzy logic model [7], decision making [8], and qualitative model [9]. Based on these models, significant research progresses on phage $\lambda$ have been made [10-15].

Most of these models contain parameters that cannot be precisely determined. For example, the model by [2] has more than 10 binding energy related parameters, each of which may vary up to $\pm 0.06 \mathrm{kcal} / \mathrm{mol}$ and thus affects system dynamics. Nonspecific binding also introduces additional factors that influence the system models [16]. Reference [17] developed a model that has 13 binding energy related parameters with uncertainty ranges around $0.5 \mathrm{kcal} / \mathrm{mol}$. Moreover, [18] pointed out that some parameters such as protein synthesis rates can be changed as surrounding environment changes. Reference [19] reported that single point mutation of DNA leads to $\pm 2 \mathrm{kcal} / \mathrm{mol}$ change for binding free energy. Due to these parameter uncertainties, it is often desired to analyze the effect of these parameters on system dynamics.

Researchers have been using sensitivity and robustness to study the influence of parameter uncertainty. Sensitivity 
and robustness are closely related because a robust system is not sensitive to parameter variations [20]. By using lysis frequency as a sensitivity measure, [21] simulated a mutation model introduced by [22] and concluded that spontaneous switching from lysogen to lysis depends sensitively on model parameters. By systematically perturbing binding energies with $\pm 1 \mathrm{kcal} / \mathrm{mol},[23$ ] concluded that lysogenic activity at promoter $P_{R M}$ remains stable under this perturbation.

In this paper we investigate bifurcation by using bistable range as a system robustness measure. In a typical case, phage $\lambda$ genetic switch model has three equilibria. Two of them are stable corresponding to the lysogenic and lytic states, and the third one is a saddle point. When some parameters are perturbed, it is possible that one stable equilibrium and the saddle point coalesce, then both of them disappear, resulting in the loss of bistable behavior. Hence, bistable region can be defined as the parameter region in which bistable behavior exists [18]. The bistable region for a parameter is large if the system dynamics is not sensitive to that parameter.

We are interested in analyzing bistable ranges for binding energy related parameters. Some other parameters such as protein degradation rate and protein synthesis rate with respect to gene transcription have already been proved that their variations may lead to the loss of bistability $[3,24]$. Our calculations are based on a phage $\lambda$ OR model [3]. Although DNA looping formed by cooperations between the left operators and the right operators affects the stability of the genetic switch [25], the OR model only includes the effects of the right operators, which are believed to play a critical role for lysogeny maintenance [26]. We will calculate bistable ranges for all binding energies, which are crucial in determining the affinity that proteins bind to phage $\lambda$ genes. Furthermore, we define Z-shaped bifurcation and calculate Jacobian matrix to study robustness. We find out that different binding energy affects lysogeny stability differently: some of them may destroy bistability, whereas others have little influence on system dynamics. Therefore, we need to precisely determine the values of those sensitive parameters in the model because otherwise we may not get an effective bistable switch.

\section{Phage $\lambda$ Modeling}

We first present phage $\lambda$ gene regulatory networks and then introduce their mathematical model. Lysogeny and lysis are two possible states for phage $\lambda$. It is believed that wild-type phage $\lambda$ has quite a stable lysogenic state since lysogeny loss rate is even lower than $10^{-8}$ per cell generation [27]. However, certain conditions such as the exposure to UV light $[28,29]$, the presence of mitomycin $C$ [30], or the starvation of the host cells [31] can induce phage $\lambda$ from lysogeny to lysis. Mathematical models have been built to successfully explain stability and high efficiency of genetic switch.

The concentrations of two proteins CI and Cro can serve as an indication of which state phage $\lambda$ is in, lysogeny or lysis. In lysogeny, the concentration of protein CI in the cell is significantly higher than that of protein Cro, whereas in lysis,

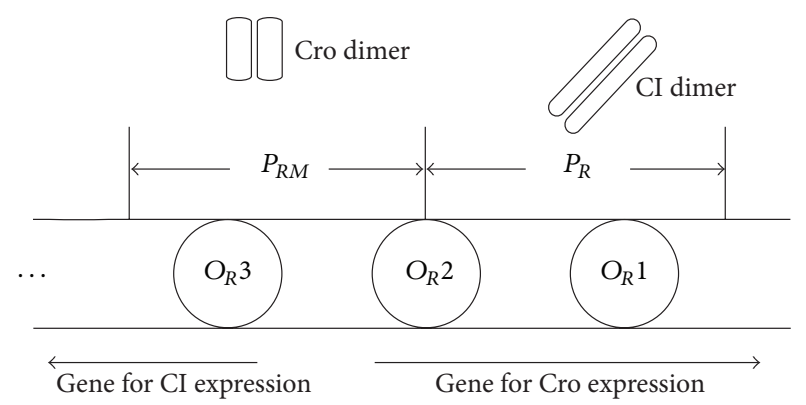

FIgure 1: Three binding sites on a DNA segment. The sites $O_{R} 1$, $O_{R} 2$, and $O_{R} 3$ can be bound by CI dimer, Cro dimer, or RNA polymerase. This DNA segment consists of promoters $P_{R M}$ and $P_{R}$. If the RNA polymerase binds to $O_{R} 3, c I$ gene is expressed; if the RNA polymerase binds to $O_{R} 1$ and $O_{R} 2$, cro gene is expressed. The amount of Cro and $\mathrm{CI}$ in the cell directly determines which state phage $\lambda$ is in.

TABLE 1: Parameter values for our model.

\begin{tabular}{lc}
\hline$\Delta G_{1 c}=-12.0 \mathrm{kcal} / \mathrm{mol}^{\dagger}$ & $\Delta G_{2 c}=-10.8 \mathrm{kcal} / \mathrm{mol}^{\dagger}$ \\
$\Delta G_{3 c}=-13.4 \mathrm{kcal} / \mathrm{mol}^{\dagger}$ & $\Delta G_{1 r}=-12.5 \mathrm{kcal} / \mathrm{mol}^{\ddagger}$ \\
$\Delta G_{2 r}=-10.5 \mathrm{kcal} / \mathrm{mol}^{\ddagger}$ & $\Delta G_{3 r}=-9.5 \mathrm{kcal} / \mathrm{mol}^{\ddagger}$ \\
$\Delta G_{12 r}=-2.7 \mathrm{kcal} / \mathrm{mol}^{\ddagger}$ & $\Delta G_{23 r}=-2.9 \mathrm{kcal} / \mathrm{mol}^{\ddagger}$ \\
$\Delta G_{R 12}=-12.5 \mathrm{kcal} / \mathrm{mol}^{\dagger}$ & $\Delta G_{R 3}=-11.5 \mathrm{kcal} / \mathrm{mol}^{\dagger}$ \\
$K_{r}=5.56 \times 10^{-9} \mathrm{M}^{\dagger}$ & $K_{c}=3.26 \times 10^{-7} \mathrm{M}^{\dagger}$ \\
$N_{\mathrm{RNAP}}=3.0 \times 10^{-8} \mathrm{M}^{\S}$ & $\mu_{1}=2.0 \times 10^{-2} \mathrm{~min}^{-1 \dagger}$ \\
$\mu_{2}=3.6 \times 10^{-2} \mathrm{~min}^{-1 \dagger}$ & $R=1.99 \mathrm{cal} /(\mathrm{mol} \cdot \mathrm{K})$ \\
$T=310 \mathrm{~K}$ & $k=1 / 11^{\Im}$ \\
\hline
\end{tabular}

${ }^{\dagger}$ denotes the parameter values from [14], ${ }^{\ddagger}$ from [32], ${ }^{\S}$ from [21], and ${ }^{g}$ from [3].

protein Cro dominates the cell. The amount of CI and Cro thus implies which state phage $\lambda$ is in.

Figure 1 illustrates the gene segment regulating cro and $c I$ gene expression. Three binding sites $O_{R} 1, O_{R} 2$, and $O_{R} 3$ control the expression of two kinds of regulatory proteins. The $P_{R M}$ promoter covers the whole binding site $O_{R} 3$ and part of $\mathrm{O}_{R} 2$, controlling $c I$ gene expression. When RNA polymerase (RNAP) binds to the site $O_{R} 3$, the gene expression is on, resulting in the production of protein CI. The $P_{R}$ promoter covers all of $O_{R} 1$ and part of $O_{R} 2$, controlling cro expression. When RNAP binds to sites $O_{R} 1$ and $O_{R} 2$ simultaneously, the cro gene expression is on. The binding sites may be occupied by either Cro or CI protein. Usually Cro and CI first form their dimers so that they are able to bind to $O_{R} 1, O_{R} 2$, and $O_{R} 3$, prohibiting the gene expressions on these sites.

Several phage $\lambda$ models have been established based on this regulating mechanism. In this paper we adopt the wellknown two-dimensional OR model stated in [3] with updated parameter values given in Table 1 . This model involves three $O_{R}$ gene binding sites, which are believed to be the most critical factors for the stability of genetic switch [33]. This two-dimensional model is in line with the general biological switch model for two-gene networks [34]. To keep it simple, we eliminate the effects from other activities in the cell, such as nonspecific binding $[16,17]$, stochastic gene expression 
[35], and intrinsic and extrinsic noises [36]. The model is described by the following system equations:

$$
\begin{aligned}
& \frac{d N_{r}}{d t}=A_{1} f_{r}-\mu_{1} N_{r}, \\
& \frac{d N_{c}}{d t}=A_{2} f_{c}-\mu_{2} N_{c},
\end{aligned}
$$

where

$$
\begin{gathered}
f_{r}=P_{28}+P_{29}+P_{30}+P_{40}, \\
f_{c}=\sum_{i=31}^{33} P_{i}+k\left(\sum_{j=34}^{40} P_{j}\right), \\
P_{s}=\frac{\exp \left(-\Delta G_{s} / R T\right) N_{r 2}^{i(s)} N_{c 2}^{j(s)} N_{\mathrm{RNAP}}^{k(s)}}{\sum_{m=1}^{40} \exp \left(-\Delta G_{m} / R T\right) N_{r 2}^{i(m)} N_{c 2}^{j(m)} N_{\mathrm{RNAP}}^{k(m)}}, \\
(s=1,2, \ldots, 40) .
\end{gathered}
$$

The subscript $s$ corresponds to 40 binding configurations shown in Table 2 . The conventions between CI dimer $\left(N_{r 2}\right)$, Cro dimer $\left(N_{c 2}\right)$, and their monomers $\left(N_{r}\right.$ and $\left.N_{c}\right)$ are

$$
\begin{aligned}
& N_{r 2}=\frac{1}{2} N_{r}+\frac{1}{8} K_{r}-\frac{1}{8} \sqrt{K_{r}^{2}+8 N_{r} K_{r}}, \\
& N_{c 2}=\frac{1}{2} N_{c}+\frac{1}{8} K_{c}-\frac{1}{8} \sqrt{K_{c}^{2}+8 N_{c} K_{c}} .
\end{aligned}
$$

From (1)-(4), $N_{r}, N_{c}$ represent concentrations of proteins $\mathrm{CI}$ and Cro. The parameters $A_{1}$ and $A_{2}$ are synthesis rates of CI and Cro, respectively; $\mu_{1}$ and $\mu_{2}$ are the overall rates that decrease protein concentration, which are caused by normal protein degradation and cell growth [37]. The function $f_{r}$ (or $f_{c}$ ) represents the overall probability that the $P_{R}$ (or $P_{R M}$ ) promoter is occupied by RNAP. $P_{s}$ is the probability that sth configuration occurs, and $\Delta G_{s}$ is the total binding energy needed for this configuration. The exponents $i(s), j(s)$, and $k(s)$ represent the numbers of CI dimers, Cro dimers, and RNAP at sth configuration, respectively. $\Delta G_{s}$ is calculated by summing up the needed single binding energies listed in Table 1, including cooperation energy. For example, $\Delta G_{5}=$ $\Delta G_{12 r}+\Delta G_{1 r}+\Delta G_{2 r}$. The left right arrows in Table 2 indicate the cooperations. $k$ is the coefficient for positive feedback. When CI dimer binds to $O_{R} 2$, positive feedback makes the transcription of $c I$ gene more efficient, hence promotes its expression. $K_{r}, K_{c}$ are dissociation constants for CI and Cro proteins, respectively. $R$ represents the universal gas constant and $T$ the absolute temperature.

\section{Bifurcation in Phage $\lambda$}

\begin{tabular}{|c|c|c|c|c|}
\hline$s$ & $\mathrm{O}_{R} 3$ & $\mathrm{O}_{R} 2$ & & $\mathrm{O}_{R} 1$ \\
\hline \multicolumn{5}{|l|}{1} \\
\hline 2 & & & & CI2 \\
\hline 3 & & $\mathrm{CI} 2$ & & \\
\hline 4 & $\mathrm{CI} 2$ & & & \\
\hline 5 & & $\mathrm{CI} 2 \leftrightarrow$ & & $\mathrm{CI} 2$ \\
\hline 6 & $\mathrm{CI} 2$ & & & $\mathrm{CI} 2$ \\
\hline 7 & $\mathrm{CI} 2 \leftrightarrow$ & $\mathrm{CI} 2$ & & \\
\hline 8 & $\mathrm{CI} 2 \leftrightarrow$ & $\mathrm{CI} 2 \leftrightarrow$ & & $\mathrm{CI} 2$ \\
\hline 9 & & & & Cro2 \\
\hline 10 & & Cro2 & & \\
\hline 11 & Cro2 & & & \\
\hline 12 & & Cro2 & & Cro2 \\
\hline 13 & Cro2 & & & Cro2 \\
\hline 14 & Cro2 & Cro2 & & \\
\hline 15 & Cro2 & Cro2 & & Cro2 \\
\hline 16 & & Cro2 & & $\mathrm{CI} 2$ \\
\hline 17 & Cro2 & & & $\mathrm{CI} 2$ \\
\hline 18 & Cro2 & Cro2 & & $\mathrm{CI} 2$ \\
\hline 19 & Cro2 & $\mathrm{CI} 2$ & & \\
\hline 20 & & $\mathrm{CI} 2$ & & Cro2 \\
\hline 21 & Cro2 & CI2 & & Cro2 \\
\hline 22 & $\mathrm{CI} 2$ & Cro2 & & \\
\hline 23 & $\mathrm{CI} 2$ & & & Cro2 \\
\hline 24 & $\mathrm{CI} 2$ & Cro2 & & Cro2 \\
\hline 25 & Cro2 & $\mathrm{CI} 2 \leftrightarrow$ & & $\mathrm{CI} 2$ \\
\hline 26 & $\mathrm{CI} 2$ & Cro2 & & $\mathrm{CI} 2$ \\
\hline 27 & $\mathrm{CI} 2 \leftrightarrow$ & $\mathrm{CI} 2$ & & Cro2 \\
\hline 28 & & & RNAP & \\
\hline 29 & $\mathrm{CI} 2$ & & RNAP & \\
\hline 30 & Cro2 & & RNAP & \\
\hline 31 & RNAP & $\mathrm{CI} 2$ & & \\
\hline 32 & RNAP & $\mathrm{CI} 2 \leftrightarrow$ & & $\mathrm{CI} 2$ \\
\hline 33 & RNAP & CI2 & & Cro2 \\
\hline 34 & RNAP & & & \\
\hline 35 & RNAP & & & $\mathrm{CI} 2$ \\
\hline 36 & RNAP & & & Cro2 \\
\hline 37 & RNAP & Cro2 & & \\
\hline 38 & RNAP & Cro2 & & $\mathrm{CI} 2$ \\
\hline 39 & RNAP & Cro2 & & Cro2 \\
\hline 40 & RNAP & & RNAP & \\
\hline
\end{tabular}

The model used in this paper is two-dimensional nonlinear ordinary differential equations. To get all the equilibria, we set the left hand side of (1) to zero and solve the transcendental equations. It can be found that in general the model has three equilibria. However, the number of equilibria may vary due to bifurcation under parameter variation. In phage $\lambda$ model,
TABLE 2: 40 Binding configurations for the three sites.

frequently occurring is the saddle node bifurcation, whose precise mathematical definition is given as follows [38].

Definition 1 (saddle node bifurcation). A saddle node bifurcation in dynamical system takes place when two equilibrium points coalesce and disappear.

In (1), two parameters $A_{1}$ and $A_{2}$ are not specified in Table 1 . They represent proportional constants relating the transcription initiation rate to the absolute rate of CI and 

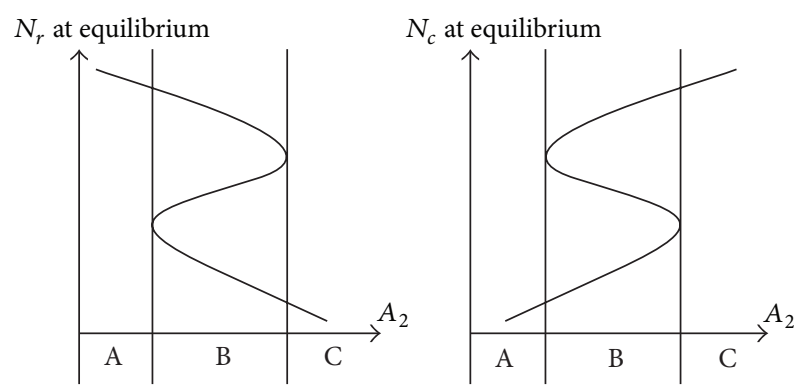

Figure 2: Saddle node bifurcation with the variation of Cro synthesis rate $A_{2}$. This is a schematic plot and not drawn to scale.

Cro protein synthesis [3]. We use Figure 2 to illustrate Zshaped bifurcation with the variation of $A_{2}$. Set $A_{1}=8 \times$ $10^{-9} \mathrm{M} / \mathrm{min}$ and divide the whole plane into three regions as shown in Figure 2, labeled by A, B, and C. Initially, when the value of $A_{2}$ is small in region $\mathrm{A}$, for example, $A_{2} \approx$ $5 \times 10^{-9} \mathrm{M} / \mathrm{min}$, there exists only one stable point and thus one single curve segment for both $N_{r}$ and $N_{c}$. At the boundary of regions $\mathrm{A}$ and $\mathrm{B}$, saddle node bifurcation occurs: $A_{2}$ increases to region $\mathrm{B}$, for example, a typical value $A_{2} \approx$ $5 \times 10^{-8} \mathrm{M} / \mathrm{min}$, and two new equilibria (one saddle and one stable equilibrium) emerge, resulting in three equilibria. Note that this is a saddle node bifurcation with the reversed direction, in comparison to Definition 1.

At the boundary of regions B and C, saddle node bifurcation takes place again, leading to the vanishing of two equilibria (one saddle point and one stable equilibrium). In region $C$, say $A_{2} \approx 5 \times 10^{-7} \mathrm{M} / \mathrm{min}$, only one stable equilibrium remains. We notice the motion of the saddle point. When the saddle point shows up at the boundary of regions $\mathrm{A}$ and $\mathrm{B}$, the lysis stable equilibrium appears as well. As parameter $A_{2}$ increases, the saddle point moves toward the lysogen stable point and finally merges with it at the boundary of regions B and C. Two successive bifurcations form a Zshaped curve are shown in Figure 2.

We have not yet specified the boundaries between these regions quantitatively. Next we solve the values of the boundaries by using the Jacobian method. Let us first introduce the following two theorems [38].

Theorem 2 (Hartman-Grobman Theorem). If the Jacobian of the nonlinear system $\dot{x}=g(x)$ at equilibrium, namely $J\left(x_{0}\right)$, has no zero or purely imaginary eigenvalues, then the phase trajectory of the system $\dot{x}=g(x)$ resembles that of the linearized system $\dot{z}=J\left(x_{0}\right) z$ near $x_{0}$ if none of the eigenvalues of $J\left(x_{0}\right)$ are on the $j \omega$ axis.

For a given equilibrium $x_{0}$, in most cases, we can determine its dynamical behavior qualitatively by calculating its Jacobian matrix $J\left(x_{0}\right)$. For a two-dimensional system, its Jacobian matrix can be calculated as

$$
J=\left[\begin{array}{ll}
\frac{\partial g_{1}}{\partial N_{r}} & \frac{\partial g_{1}}{\partial N_{c}} \\
\frac{\partial g_{2}}{\partial N_{r}} & \frac{\partial g_{2}}{\partial N_{c}}
\end{array}\right] .
$$

The following theorem gives the necessary conditions for the critical point that bifurcation occurs [38].

Theorem 3. For any nonlinear system $\dot{z}=g(z, \mu)$, the system goes through a saddle node bifurcation at $z=z^{\dagger}, \mu=\mu^{\dagger}$ only if the following relations are satisfied:

$$
\begin{gathered}
g\left(z^{\dagger}, \mu^{\dagger}\right)=0, \\
\left|\frac{\partial g}{\partial z}\left(z^{\dagger}, \mu^{\dagger}\right)\right|=0 .
\end{gathered}
$$

From (6), $z^{\dagger}$ is an equilibrium point. In a planar system, (7) is the determinant of system Jacobian. When the determinant of Jacobian matrix vanishes, at least one eigenvalue is zero. Jacobian is a characterization of planar system dynamics. For variation of parameter $A_{2}$, the system in (1) can be rewritten as

$$
\begin{aligned}
& \frac{d N_{r}}{d t}=g_{1}\left(N_{c}, N_{r}, A_{2}\right), \\
& \frac{d N_{c}}{d t}=g_{2}\left(N_{c}, N_{r}, A_{2}\right) .
\end{aligned}
$$

From Theorems 2 and 3 , for a given $A_{2}$, the Jacobian matrix is evaluated at equilibrium points $N_{c}^{\dagger}$ and $N_{r}^{\dagger}$. These two equilibrium points can be represented as a function of $A_{2}$ as shown in Figure 2.

By Theorem 3, we know that the bifurcation appears when Jacobian has a zero eigenvalue. From Figure 3, we can obtain the approximate values of critical points by checking the points of the eigenvalue curves crossing zero line. These values are $5.6 \times 10^{-9} \mathrm{M} / \mathrm{min}$ and $3.4 \times 10^{-7} \mathrm{M} / \mathrm{min}$.

To determine the stability of an equilibrium point, we calculate the eigenvalue of the Jacobian matrix. Figure 3 plots the eigenvalues as a function of $A_{2}$. The Jacobian matrix can be calculated analytically (see the Appendix for details). By solving the value of $A_{2}$ that makes the determinant of the Jacobian matrix vanish, we get the critical value of $A_{2}$ at which bifurcation occurs. When $A_{2}$ is small (less than $5.6 \times 10^{-9} \mathrm{M} / \mathrm{min}$ in Figure 3), there are only two negative eigenvalues corresponding to one equilibrium point. This equilibrium is thus stable [38].

As $A_{2}$ exceeds $5.6 \times 10^{-9} \mathrm{M} / \mathrm{min}$, we have the case when there are three equilibria and correspondingly six eigenvalues. Among these three equilibria, two are stable and the third is unstable (saddle point). This is because positive eigenvalue appears at saddle point (see dash-dot line in Figure 3). For a two-dimensional linear system, if the Jacobian has one positive and one negative eigenvalues at the equilibrium, the equilibrium is a saddle point. If $A_{2}$ continues increasing, only one equilibrium point is left $\left(A_{2}\right.$ greater than $3.4 \times 10^{-9} \mathrm{M} / \mathrm{min}$ in this case), and both of its eigenvalues are negative. The curves in Figure 3 have two intersections with zero eigenvalue, which indicates that the eigenvalues of Jacobian vanish twice, forming a Z-shaped bifurcation. 


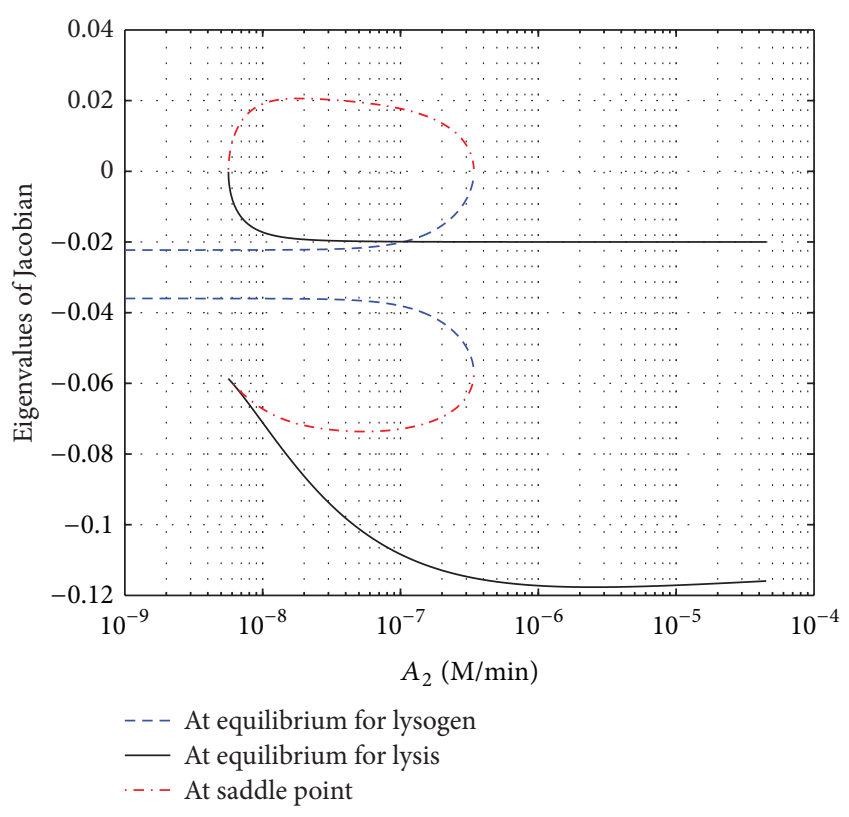

FIGURE 3: Jacobian eigenvalues at equilibria. The figure shows how the eigenvalues of Jacobian at equilibria change with $A_{1}$ fixed at $8 \times 10^{-9} \mathrm{M} / \mathrm{min}$ and $A_{2}$ varying. The solid line (black) shows the eigenvalues of lysis equilibrium. The dashed line (blue) is for lysogeny equilibrium. The dot-dashed line (red) is for saddle.

\section{Bifurcation Analysis of Binding Energy Uncertainty}

In this section, we will analyze bifurcation for $\Delta G$ 's by using the Jacobian method. In Table 1, the symbol $\Delta G$ with subscripts such as $\Delta G_{1 r}$ and $\Delta G_{2 c}$ represents Gibbs free energy required for $\mathrm{CI}$ or Cro dimers to bind to the OR sites. Because of the difficulty in determining the precise values of the binding energy, it is useful to study how the equilibria change as these Gibbs free binding energies vary. In this section, we set synthesis rates $A_{1}$ and $A_{2}$ at appropriate values, $A_{1}=8 \times 10^{-9} \mathrm{M} / \mathrm{min}$ and $A_{2}=5 \times 10^{-8} \mathrm{M} / \mathrm{min}$. To calculate the equilibria, we set the left hand side of (1) to zero and obtain the expression of $N_{r}$ and $N_{c}$ as functions of $\Delta G$ 's:

$$
\begin{aligned}
& N_{r}^{*}=f_{1}\left(\Delta G_{1 c}, \Delta G_{2 c}, \ldots, \Delta G_{R 3}\right), \\
& N_{c}^{*}=f_{2}\left(\Delta G_{1 c}, \Delta G_{2 c}, \ldots, \Delta G_{R 3}\right),
\end{aligned}
$$

where $N_{r}^{*}$ and $N_{c}^{*}$ are the concentrations of proteins $\mathrm{CI}$ and Cro at the equilibrium point. Once (9) is obtained, we can determine the quantitative behavior around equilibria when changing $\Delta G$ 's. The relative sensitivities are given by

$$
\begin{gathered}
S_{N_{r}}\left(\Delta G_{s}\right)=\frac{\partial f_{1}}{\partial \Delta G_{s}}, \\
S_{N_{c}}\left(\Delta G_{s}\right)=\frac{\partial f_{2}}{\partial \Delta G_{s}},
\end{gathered}
$$

where the subscript $s \in\{1 r, 2 r, \ldots, R 12, R 3\}$. Since the system equilibria cannot be analytically solved, we may apply the numerical procedure to calculate (10) as a measure of parameter sensitivity. As to the study on system robustness, the Jacobian method can be used to study bifurcation introduced by parameter variation.

Since there are ten different $\Delta G s$, we divide them into four groups:

(1) $\Delta G_{1 r}, \Delta G_{2 r}$, and $\Delta G_{3 r}$ : binding free energies of CI;

(2) $\Delta G_{1 c}, \Delta G_{2 c}$, and $\Delta G_{3 c}$ : binding free energies of Cro;

(3) $\Delta G_{12 r}$ and $\Delta G_{23 r}$ : the cooperation between proteins CI's if both $O_{R} 1$ and $O_{R} 2$, or $O_{R} 2$ and $O_{R} 3$ are bound by CI dimers;

(4) $\Delta G_{R 12}$ and $\Delta G_{R 3}$ : RNAP binding energies.

We then study each of these four groups.

4.1. $\Delta G_{1 r}, \Delta G_{2 r}$, and $\Delta G_{3 r}$. We systematically perturb the values of $\Delta G_{1 r}, \Delta G_{2 r}$, and $\Delta G_{3 r}$ to investigate their impact on the system dynamics. Two proper robustness measures are the range of parameters in which lysogeny exists (stable range), and in which bistable dynamics exists (bistable range). In stable range, it is possible to keep phage $\lambda$ dormant and integrate into the gene of the host. On the other hand, bistable range defines the parameter range to keep bistability and the maximal uncertainty tolerance. In this case, it implies that the system favors dynamics with two stable equilibria and a saddle. Further, if the stochastic force is strong enough, phage $\lambda$ can switch from the lysogenic stable point to the lytic stable point even without the parameter changes.

Figures 4(a)-4(c) illustrate the impact of $\Delta G_{1 r}, \Delta G_{2 r}$, and $\Delta G_{3 r}$ variations. We set parameter values as in Table 1 and find out all the equilibria when varying one $\Delta G_{s}$. We find that the energy $\Delta G_{3 r}$ does not show Z-shaped bifurcation, while the other two parameters $\Delta G_{1 r}$ and $\Delta G_{2 r}$ show quite similar behaviors since Figures $4(\mathrm{a})$ and $4(\mathrm{~b})$ are almost identically the same. The result is not surprising because $\Delta G_{1 r}$ and $\Delta G_{2 r}$ play similar roles in system dynamics. These two parameters determine the likelihood that CI dimer binds to sites $O_{R} 1$ and $O_{R} 2$. When either of the two sites is occupied by protein dimers, the expression of cro gene is prohibited, resulting in lysogenic state. Nonetheless, the parameter $\Delta G_{3 r}$ indicates the likelihood of CI dimer binding to the site $O_{R} 3$. If the absolute value of $\Delta G_{3 r}$ is too large, it is easy for CI dimer to bind to $\mathrm{O}_{R} 3$; thus, $c I$ gene expression is prohibited, resulting in lysis. If the absolute value of $\Delta G_{3 r}$ is small, the $Z$-shaped bifurcation vanishes. In this case, $c I$ gene expression is not prohibited, but it has no influence on cro gene expression. Bistable behavior always exists when $\Delta G_{3 r}$ is less than its nominal value in Table 1. Without stopping or weakening the expression of cro gene, single lysogen stable point layout cannot be achieved just by increasing $\Delta G_{3 r}$. Compared with other parameters, the system dynamics is more robust to $\Delta G_{3 r}$ variation.

Table 3 lists the stable range, bistable range, and the length of bistable range for all ten $\Delta G$ s. It can be shown by Theorem 2 that one of the three equilibria is a saddle and the other two are stable points. Jacobian matrix is also used to determine the boundary of stable and bistable ranges according to Theorem 3. As long as $\Delta G_{1 r}$ is less than the nominal value 


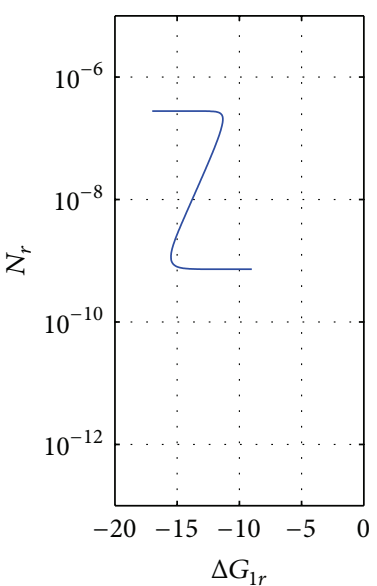

(a) Z-shaped bifurcation for $\Delta G_{1 r}$

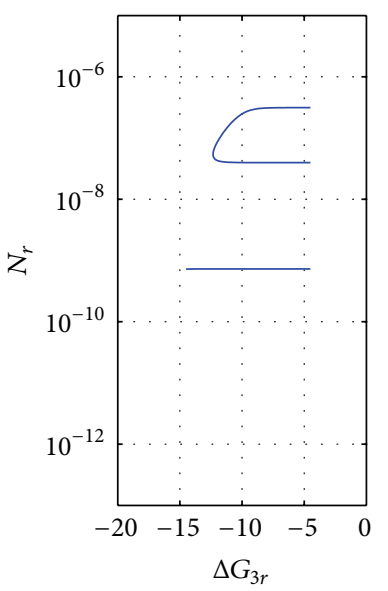

(c) Bifurcation for $\Delta G_{3 r}$

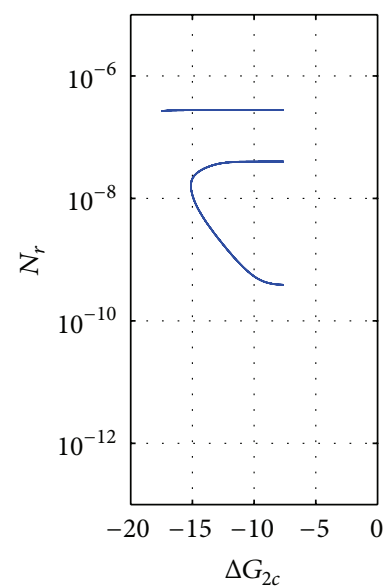

(e) Bifurcation for $\Delta G_{2 c}$
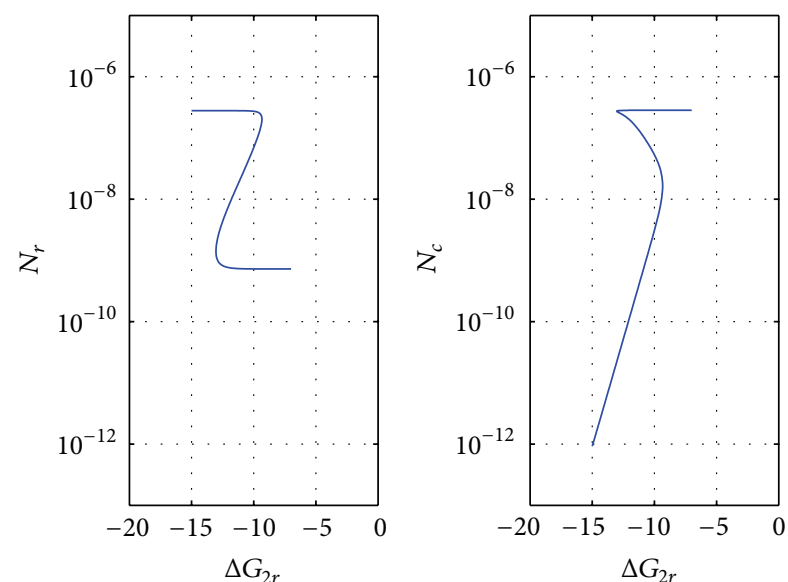

(b) Z-shaped bifurcation for $\Delta G_{2 r}$
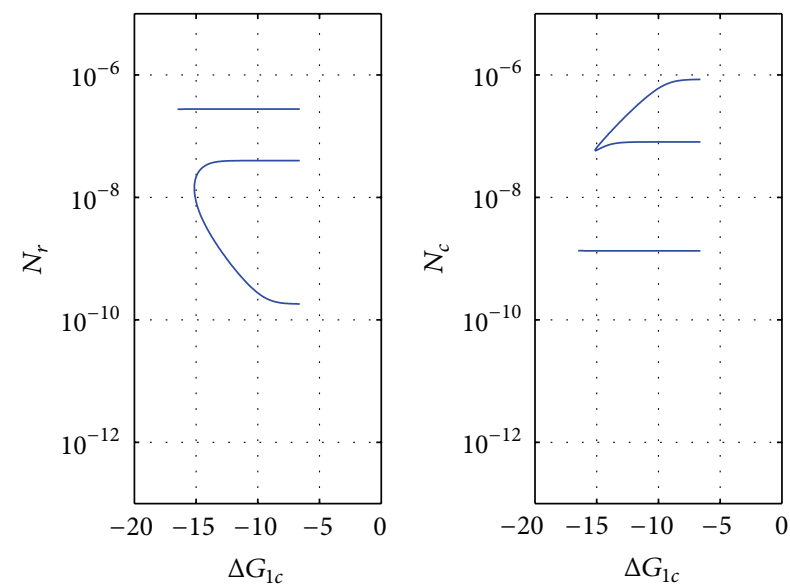

(d) Bifurcation for $\Delta G_{1 c}$
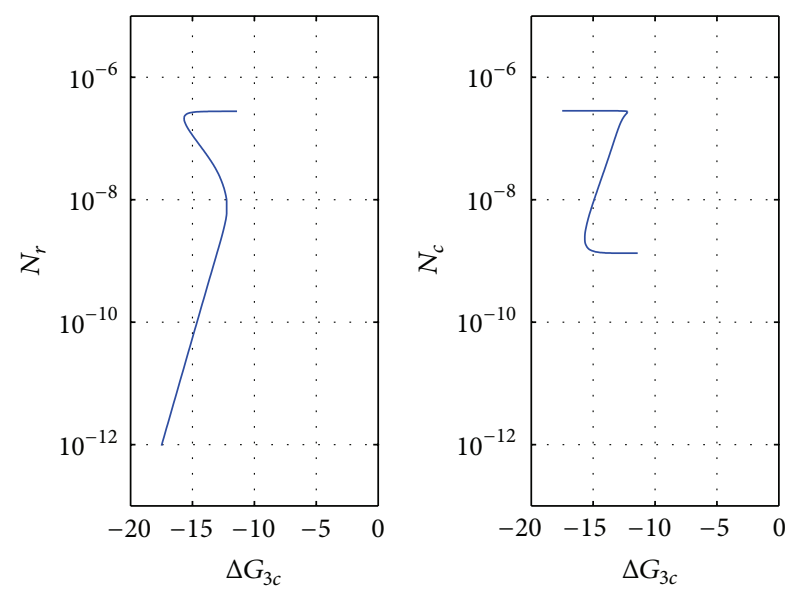

(f) Z-shaped bifurcation for $\Delta G_{3 c}$

FIGURE 4: Movement of equilibria under parameter variation. Z-shaped bifurcation can be generated by variation of $\Delta G_{1 r}, \Delta G_{2 r}$, and $\Delta G_{3 c}$. All vertical axes are plotted on a log scale.

at $-11.33 \mathrm{kcal} / \mathrm{mol}$, or $\Delta G_{2 r}$ is less than $-9.34 \mathrm{kcal} / \mathrm{mol}$, the system always has lysogenic state. They are critical bifurcation points. For $\Delta G_{3 r}$, however, lysogenic state always exists if it is greater than $-12.33 \mathrm{kcal} / \mathrm{mol}$. Consequently, for CI protein, a larger binding energy to $O_{R} 1$ and $O_{R} 2$ helps to keep the DNA of phage $\lambda$ silent in the host. The system tends to be unstable if the values of binding energies for CI to $O_{R} 1$ and $O_{R} 2$ become small, thus forming Z-shaped bifurcation. Parameter variations may lead to $Z$-shaped bifurcation if and only if the bistable range is bounded. 
TABLE 3: Robustness measures of binding energies.

\begin{tabular}{lccc}
\hline Variable & $\begin{array}{c}\text { Stable Range } \\
(\mathrm{kcal} / \mathrm{mol})\end{array}$ & $\begin{array}{c}\text { Bistable Range } \\
(\mathrm{kcal} / \mathrm{mol})\end{array}$ & $\begin{array}{c}\text { Length } \\
(\mathrm{kcal} / \mathrm{mol})\end{array}$ \\
\hline$\Delta G_{1 r}$ & $(-\infty,-11.33)$ & $(-15.49,-11.33)$ & 4.16 \\
$\Delta G_{2 r}$ & $(-\infty,-9.34)$ & $(-13.04,-9.34)$ & 3.70 \\
$\Delta G_{3 r}$ & $(-12.33,+\infty)$ & $(-12.33,+\infty)$ & $\infty$ \\
$\Delta G_{1 c}$ & $(-\infty,+\infty)$ & $(-15.14,+\infty)$ & $\infty$ \\
$\Delta G_{2 c}$ & $(-\infty,+\infty)$ & $(-15.12,+\infty)$ & $\infty$ \\
$\Delta G_{3 c}$ & $(-15.67,+\infty)$ & $(-15.67,-12.24)$ & 3.43 \\
$\Delta G_{12 r}$ & $(-\infty,-1.55)$ & $(-6.03,-1.55)$ & 4.48 \\
$\Delta G_{23 r}$ & $(-7.71, \infty)$ & $(-7.71,+\infty)$ & $\infty$ \\
$\Delta G_{R 12}$ & $(-13.67, \infty)$ & $(-13.67,-10.86)$ & 2.81 \\
$\Delta G_{R 3}$ & $(-\infty,-10.50)$ & $(-12.66,-10.50)$ & 2.16 \\
\hline
\end{tabular}

When the stochastic noise is strong enough, the noise may induce the system to lytic state. Moreover, if free energy varies due to gene mutation, bistable behavior might be destroyed and only one stable equilibrium is left. This is what the mathematical model implies about the parameter influence on the system dynamics.

4.2. $\Delta G_{1 c}, \Delta G_{2 c}$, and $\Delta G_{3 c}$. The bifurcation results of $\Delta G_{1 c}$, $\Delta G_{2 c}$, and $\Delta G_{3 c}$ are shown in Figures $4(\mathrm{~d})-4(\mathrm{f})$. The equilibria behaviors with respect to $\Delta G_{1 c}$ and $\Delta G_{2 c}$ variation are similar to $\Delta G_{3 r} . \Delta G_{1 c}$ represents the likelihood that Cro dimer binds to site $O_{R} 1$. If Cro dimer binds to site $O_{R} 1$, the expression of cro is prohibited, resulting in only one single lysogenic stable point. When $\Delta G_{1 c}$ or $\Delta G_{2 c}$ is small, the prohibition effect is weak, but this is not enough to drive the system dynamics to only one single lysis stable point. Hence, bistable layout always exists if these two parameters are small. The influence of $\Delta G_{3 c}$ is similar to the influence of $\Delta G_{1 r}$ and $\Delta G_{2 r}$. The bistable range of $\Delta G_{3 c}$ is about $3.43 \mathrm{kcal} / \mathrm{mol}$, which is comparable to those of $\Delta G_{1 r}$ and $\Delta G_{2 r}$.

The six parameters we have shown so far are the basic binding energies. The values of $\Delta G_{3 r}, \Delta G_{1 c}$, and $\Delta G_{2 c}$ decide the likelihood that negative feedback (i.e., self-prohibition) happens. It is known that the sites $O_{R} 1$ and $O_{R} 2$ are used to transcript and then translate protein Cro. The site $O_{R} 3$ contributes to the production of protein CI. If protein CI binds to $O_{R} 3$, or Cro binds to $O_{R} 1$ and $O_{R} 2$, they can stop their own transcription. Thus, we call the phenomenon negative feedback. Figure 4 shows that such negative feedback cannot take the system to Z-shaped bifurcation. The negative feedback occurs when the amount of Cro or CI is too large. For instance, the binding between $\mathrm{CI}$ dimer and $\mathrm{O}_{R} 3$ readily occurs when the amount of protein $\mathrm{CI}$ is too large. On the other hand, $\Delta G_{1 r}, \Delta G_{2 r}$, and $\Delta G_{3 c}$ represent the likelihood of positive feedback (i.e., mutual prohibition). For instance, with large $\Delta G_{3 c}$, the binding between Cro and $O_{R} 3$ is favored, stopping the expression of $c I$ gene. If the value of $\Delta G_{3 r}$ is small, and the negative feedback is weakened, but it is not enough to make the system dynamics change from single lysis equilibrium to single lysogenic equilibrium. Parameters associated with positive feedback can show Z-shaped bifurcation, whereas parameters associated with negative feedback
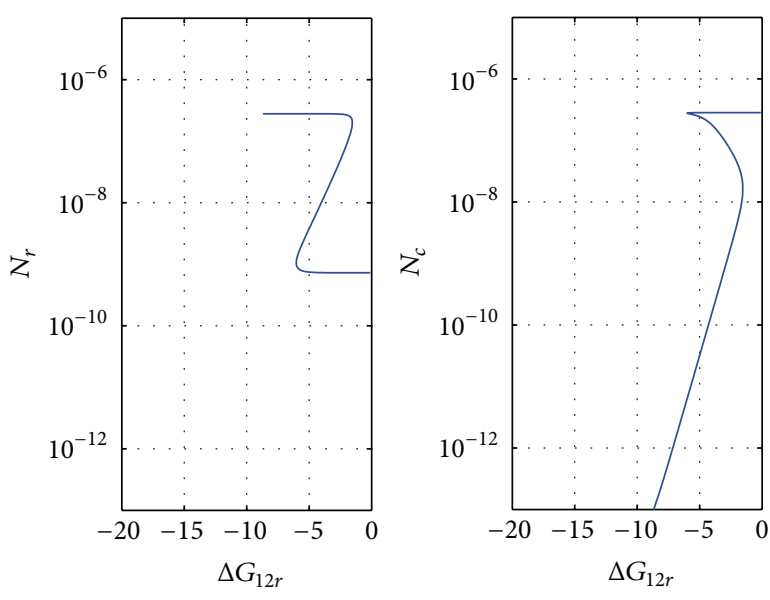

(a) Z-shaped bifurcation for $\Delta G_{12 r}$
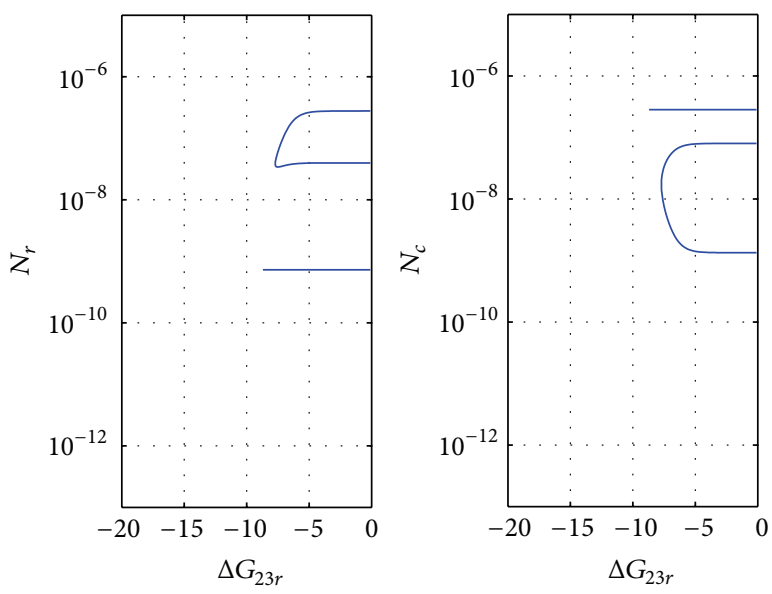

(b) Bifurcation for $\Delta G_{23 r}$

FIGURE 5: Z-shaped bifurcation generated by variation of $G_{12 r}$ and $G_{23 r}$. The variation of $\Delta G_{12 r}$ also leads to Z-shaped bifurcation, while $\Delta G_{23 r}$ is not sensitive enough to create $Z$-shaped bifurcation.

cannot. Hence, the system is more robust to negative feedback parameters than to positive feedback parameters.

4.3. $\Delta G_{12 r}$ and $\Delta G_{23 r}$. The parameters $\Delta G_{12 r}$ and $\Delta G_{23 r}$ determine the energy difference caused by the cooperation of adjacent binding CI dimers. Although [32] discovered that adjacent Cro dimers also have cooperations, we ignore this cooperation effect since it has a small cooperation energy and focus on CI cooperation in our analysis. Figure 5 shows the bifurcation results for the parameters $\Delta G_{12 r}$ and $\Delta G_{23 r}$. The nominal values of $\Delta G_{12 r}$ and $\Delta G_{23 r}$ are close to zero. Since protein cooperation process is an exothermic reaction, $\Delta G_{12 r}$ and $\Delta G_{23 r}$ must be less than zero. We find out that the variation of $\Delta G_{12 r}$ leads to $Z$-shaped bifurcation whereas $\Delta G_{23 r}$ does not. The value of $\Delta G_{23 r}$ affects the expression of both cro and $c I$ genes, whereas $\Delta G_{12 r}$ only promotes mutual prohibition from CI to Cro. $\Delta G_{23 r}$ with large value prohibits the binding of RNAP to both sites $O_{R} 2$ and $O_{R} 3$, thus both $c I$ and cro gene expressions are closed. 

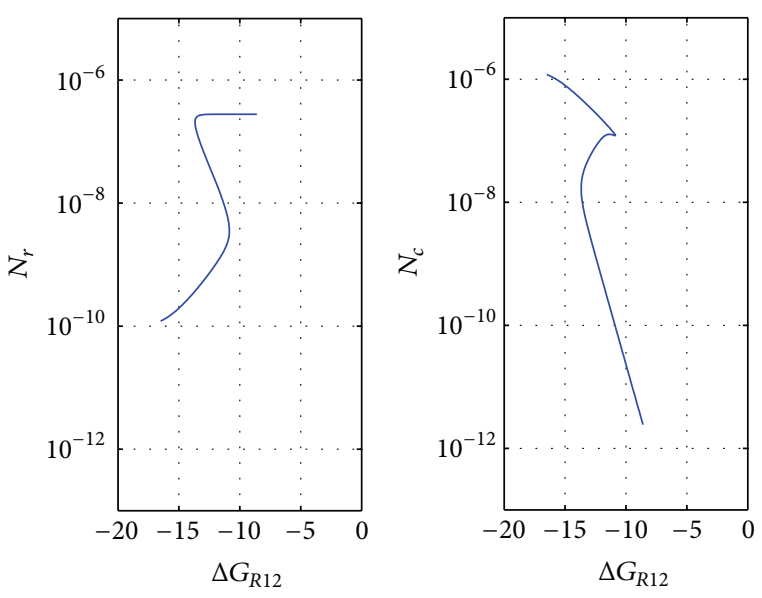

(a) Z-shaped bifurcation for $\Delta G_{R 12}$
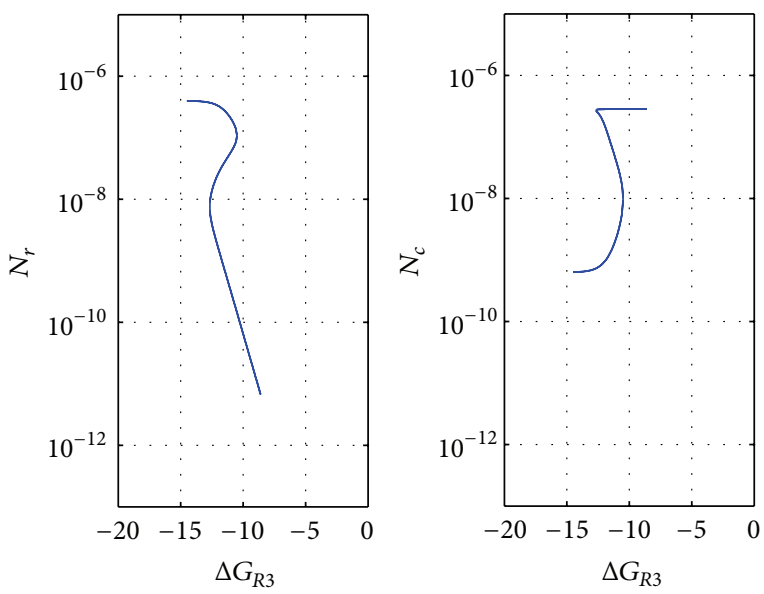

(b) Z-shaped bifurcation for $\Delta G_{R 3}$

FIGURE 6: Z-shaped bifurcation generated by variation of $G_{R 12}$ and $G_{R 3}$. Both of them are able to generate Z-shaped bifurcation. Their bistable ranges are obviously smaller than any other parameters. The appearance of "Z" shows steep slope.

Moreover, we know the length of bifurcation range for $\Delta G_{12 r}$ is $4.48 \mathrm{kcal} / \mathrm{mol}$, which is the maximal value among all the finite values in Table 3. Compared with the influence from other parameters, the system is relatively more robust to the cooperation energy variations. The bistable range for $\Delta G_{12 r}$ is from $-6.03 \mathrm{kcal} / \mathrm{mol}$ to $-1.55 \mathrm{kcal} / \mathrm{mol}$ (Table 3 ). In our model, if $\Delta G_{12 r}$ is equal to the center value at $-3.79 \mathrm{kcal} / \mathrm{mol}$, the system is most robust to parameter uncertainty because of maximum tolerance of uncertainty. This result is consistent with the calculation by [39], where they calculated biological behaviors in phage $\lambda$ and found that cooperation energy is $-3.7 \mathrm{kcal} / \mathrm{mol}$, in comparison to the in vitro value $-2.7 \mathrm{kcal} / \mathrm{mol}$ used in this work. The $1.0 \mathrm{kcal} / \mathrm{mol}$ difference between these two values might be caused by the looping effect [40]. Hence, the looping effect makes the system more robust to $\Delta G_{12 r}$ from this point of view.

4.4. $\Delta G_{R 12}$ and $\Delta G_{R 3}$. Figure 6 illustrates the influence of $\Delta G_{R 3}$ and $\Delta G_{R 12}$ variations. From Figure 6, we find that Zshaped bifurcation appears for both parameters. Note that the appearance of $\mathrm{Z}$ is different from the previous parameters as discussed above. As in Table 3, the length of bistable range for $\Delta G_{R 12}$ is $2.81 \mathrm{kcal} / \mathrm{mol}$, and for $\Delta G_{R 3}$ is only $2.16 \mathrm{kcal} / \mathrm{mol}$, which is the minimum among all ten parameters.

Notice that $\Delta G_{R 3}$ and $\Delta G_{R 12}$ are the binding free energies of RNAP to $P_{R M}$ promoter $\left(O_{R} 3\right)$ and to $P_{R}$ promoter $\left(O_{R} 1\right.$ and $O_{R} 2$ ). Because RNAP is necessary for gene expression, the result from RNAP could be the most influential. Table 3 summarizes all the sensitivity measures for the above ten parameters. We observe that the last two parameters have the shortest length of bistable ranges, which implies that bistable behavior of phage $\lambda$ is more sensitive to $\Delta G_{R 12}$ and $\Delta G_{R 3}$ than any other parameters. For example, the bistable ranges of $\Delta G_{1 r}$ and $\Delta G_{2 r}$ are around $4 \mathrm{kcal} / \mathrm{mol}$, while those of $\Delta G_{R 12}$ and $\Delta G_{R 3}$ are less than $3 \mathrm{kcal} / \mathrm{mol}$. Parameter uncertainties of $\Delta G_{R 12}$ and $\Delta G_{R 3}$ most easily destroy bistable behavior. The concentrations of $\mathrm{CI}$ and $\mathrm{Cro}$ at equilibrium point also change significantly under $\Delta G_{R 12}$ or $\Delta G_{R 3}$ uncertainty.

4.5. Discussion. From the above analysis, we find that the binding free energies to sites $O_{R} 1$ and $O_{R} 2$ have similar effects on system dynamics. As we have shown in Table 3 , the results of $\Delta G_{1 r}, \Delta G_{2 r}, \Delta G_{1 c}$, and $\Delta G_{2 c}$ are similar. This is mainly because of the similar status for binding sites $O_{R} 1$ and $O_{R} 2$, both of which control the expression of Cro protein.

Moreover, the bistable ranges have infinite length for $\Delta G_{3 r}, \Delta G_{1 c}, \Delta G_{2 c}$, and $\Delta G_{23 r}$. All of the above four parameters do not have Z-shaped bifurcation. When the values of those parameters become close to zero, bistable behavior always exists. The parameters $\Delta G_{1 c}$ and $\Delta G_{2 c}$ can be used to maintain lysogenic state when they are less than a critical value because their stable ranges do not have any limits. For example, if we introduce gene mutation to make $\Delta G_{1 c}$ lower than $-15.14 \mathrm{kcal} / \mathrm{mol}$, bistable behavior disappears and only one lysogenic state is left. Consequently, the state of phage $\lambda$ stabilizes at lysogeny.

As to the other six parameters with limited bistable ranges in Table 3, their variations lead to Z-shaped bifurcations. We are interested in why the original system seems more sensitive to these six parameters. The free energies $\Delta G_{1 r}$, $\Delta G_{2 r}$, and $\Delta G_{12 r}$ indicate the likelihood of protein CI binding to sites $O_{R} 1$ and $O_{R} 2$, or the likelihood of mutual prohibition occurring. Similarly, $\Delta G_{3 c}$ indicates the likelihood of protein Cro binding to site $O_{R} 3$ and also results in mutual prohibition. Our result has shown that, compared with selfprohibition, the mutual prohibition is more influential on system dynamics. The binding energy uncertainty related to mutual prohibition may lead to the loss of bistable behavior.

Note that the system dynamics of this model is even more sensitive to the parameters $\Delta G_{R 12}$ and $\Delta G_{R 3}$. Since $\Delta G_{R 12}$ and $\Delta G_{R 3}$ have the minimum bistable ranges, the uncertainties of these two parameters are more likely to lead to the loss of bistable behavior. This is consistent with the conclusion by [23] that perturbation of RNAP binding energy significantly changes promoter activity, thus affecting gene expression. The binding free energies of RNAP to cro and $c I$ promoters play a key role for the generation of proteins. 
In building mathematical models for phage $\lambda$ genetic switch, it is crucial to determine the values for those parameters that are more sensitive to parameter uncertainties such as $\Delta G_{R 12}$ and $\Delta G_{R 3}$, because otherwise the system dynamics may deviate from normal bistable behavior. Furthermore, our results provide guidelines for the experimentalists in laboratory on how to choose those parameters that can alter mutations more easily.

\section{Conclusion}

In this paper we studied the robustness of a phage $\lambda$ model by investigating its bifurcation behavior induced by parameter uncertainties. We introduced bistable range as a measure of robustness and then applied the Jacobian method to calculate it. It can be concluded that binding energy uncertainties may destroy bistable behavior, especially for those sensitive parameters such as $\Delta G_{R 12}$ and $\Delta G_{R 3}$. Moreover, parameters describing mutual prohibition can form $Z$-shaped bifurcation (i.e., $\Delta G_{1 r}, \Delta G_{2 r}, \Delta G_{3 c}$, and $\Delta G_{12 r}$ ), whereas parameters describing self-prohibition (i.e., $\Delta G_{3 r}, \Delta G_{1 c}$, and $\Delta G_{2 c}$ ) cannot. Hence, system dynamics is more sensitive to mutual prohibition parameters.

There also exist other factors affecting the stability and robustness of a phage $\lambda$ model. For example, DNA looping can promote CI expression in lysogenic state [14] and thus help phage $\lambda$ keep stable and robust [25]. Our future work includes exploring the bifurcation and system robustness with respect to these new factors.

\section{Appendix}

\section{Calculation of Jacobian}

From (1)-(5), we may represent system Jacobian by

$$
J=\left[\begin{array}{cc}
A_{1} \frac{\partial P_{r}}{\partial N_{r}}-\mu_{1} & A_{1} \frac{\partial P_{r}}{\partial N_{c}} \\
A_{2} \frac{\partial P_{c}}{\partial N_{r}} & A_{2} \frac{\partial P_{c}}{\partial N_{c}}-\mu_{2}
\end{array}\right] .
$$

Since $P_{r}$ and $P_{c}$ are sums of $P_{s}, s=1,2, \ldots, 40$, as (2) shows, the key part to evaluate their partial derivatives in (A.1) is $\partial P_{s} / \partial N_{r}$ and $\partial P_{s} / \partial N_{c}$. Since $P_{s}$ is a function with respect to Cro and CI dimers, we evaluate the partial derivatives by Chain Rule:

$$
\begin{gathered}
\frac{\partial P_{s}}{\partial N_{r}}=\frac{\partial P_{s}}{\partial N_{r 2}} \frac{d N_{r 2}}{d N_{r}}, \\
\frac{\partial P_{s}}{\partial N_{c}}=\frac{\partial P_{s}}{\partial N_{c 2}} \frac{d N_{c 2}}{d N_{c}},
\end{gathered}
$$

\begin{tabular}{|c|c|c|c|}
\hline$s$ & $i(s)$ & $j(s)$ & $k(s)$ \\
\hline 1 & 0 & 0 & 0 \\
\hline 2 & 1 & 0 & 0 \\
\hline 3 & 1 & 0 & 0 \\
\hline 4 & 1 & 0 & 0 \\
\hline 5 & 2 & 0 & 0 \\
\hline 6 & 2 & 0 & 0 \\
\hline 7 & 2 & 0 & 0 \\
\hline 8 & 3 & 0 & 0 \\
\hline 9 & 0 & 1 & 0 \\
\hline 10 & 0 & 1 & 0 \\
\hline 11 & 0 & 1 & 0 \\
\hline 12 & 0 & 2 & 0 \\
\hline 13 & 0 & 2 & 0 \\
\hline 14 & 0 & 2 & 0 \\
\hline 15 & 0 & 3 & 0 \\
\hline 16 & 1 & 1 & 0 \\
\hline 17 & 1 & 1 & 0 \\
\hline 18 & 1 & 2 & 0 \\
\hline 19 & 1 & 1 & 0 \\
\hline 20 & 1 & 1 & 0 \\
\hline 21 & 1 & 2 & 0 \\
\hline 22 & 1 & 1 & 0 \\
\hline 23 & 1 & 1 & 0 \\
\hline 24 & 1 & 2 & 0 \\
\hline 25 & 2 & 1 & 0 \\
\hline 26 & 2 & 1 & 0 \\
\hline 27 & 1 & 2 & 0 \\
\hline 28 & 0 & 0 & 1 \\
\hline 29 & 1 & 0 & 1 \\
\hline 30 & 0 & 1 & 1 \\
\hline 31 & 1 & 0 & 1 \\
\hline 32 & 2 & 0 & 1 \\
\hline 33 & 1 & 1 & 1 \\
\hline 34 & 0 & 0 & 1 \\
\hline 35 & 1 & 0 & 1 \\
\hline 36 & 0 & 1 & 1 \\
\hline 37 & 0 & 1 & 1 \\
\hline 38 & 1 & 1 & 1 \\
\hline 39 & 0 & 2 & 1 \\
\hline 40 & 0 & 0 & 2 \\
\hline
\end{tabular}

TABLE 4: Exponents for 30 binding configurations in Table 2.

where

$$
\begin{aligned}
& \frac{d N_{r 2}}{d N_{r}}=\frac{1}{2}\left(1-\frac{K_{r}}{\sqrt{K_{r}^{2}+8 N_{r} K_{r}}}\right), \\
& \frac{d N_{c 2}}{d N_{c}}=\frac{1}{2}\left(1-\frac{K_{c}}{\sqrt{K_{c}^{2}+8 N_{c} K_{c}}}\right) .
\end{aligned}
$$


Then we have to deal with other terms in (A.2). Note that $P_{s}$ is a fractional function in (3), so we write its numerator $A_{s}$ and denominator $B$ as

$$
\begin{gathered}
M_{s}=\exp \left(-\frac{\Delta G_{s}}{R T}\right) N_{r 2}^{i(s)} N_{c 2}^{j(s)} N_{\mathrm{RNAP}}^{k(s)}, \\
D=\sum_{s=1}^{40} M_{s} .
\end{gathered}
$$

The partial derivatives for $P_{s}$ with respect to $N_{c 2}$ and $N_{r 2}$ are given by

$$
\begin{gathered}
\frac{\partial P_{s}}{\partial N_{r 2}}=\frac{\partial M_{s}}{\partial N_{r 2}} \frac{1}{D}-\frac{\partial D}{\partial N_{r 2}} \frac{M_{s}}{D^{2}}, \\
\frac{\partial P_{s}}{\partial N_{c 2}}=\frac{\partial M_{s}}{\partial N_{c 2}} \frac{1}{D}-\frac{\partial D}{\partial N_{c 2}} \frac{M_{s}}{D^{2}} .
\end{gathered}
$$

The partial derivative of $M_{s}$ can be obtained as

$$
\begin{aligned}
& \frac{\partial M_{s}}{\partial N_{r 2}}=i(s) \exp \left(-\frac{\Delta G_{s}}{R T}\right) N_{r 2}^{i(s)-1} N_{c 2}^{j(s)} N_{\mathrm{RNAP}}^{k(s)}, \\
& \frac{\partial M_{s}}{\partial N_{c 2}}=j(s) \exp \left(-\frac{\Delta G_{s}}{R T}\right) N_{r 2}^{i(s)} N_{c 2}^{j(s)-1} N_{\mathrm{RNAP}}^{k(s)} .
\end{aligned}
$$

The subscript $s$ corresponds to the 40 binding configurations. The partial derivative of $D$ is exactly the sum of $40 M_{s}$ partial derivatives. From the above derivation, we may obtain the Jacobian matrix analytically. This is quite helpful when we calculate the eigenvalues of Jacobian matrix at equilibrium points. By referencing Table 2, we may count the exponents (i.e., $i(s), j(s)$, and $k(s))$ in (A.4) and list them in Table 4.

\section{Conflict of Interests}

The authors declare that there is no conflict of interests regarding the publication of this paper.

\section{Acknowledgments}

This research has been supported by the China National 973 Project under Grant no. 2010CB529200, the Innovation Program of Shanghai Municipal Education Commission under Grant no. 11ZZ20, the Shanghai Pujiang Program under Grant no. 11PJ1405800, NSFC under Grant no. 61174086, and Project-sponsored by SRF for ROCS SEM.

\section{References}

[1] G. K. Ackers, A. D. Johnson, and M. A. Shea, "Quantitative model for gene regulation by $\lambda$ phage repressor," Proceedings of the National Academy of Sciences of the United States of America, vol. 79, no. 4 I, pp. 1129-1133, 1982.

[2] M. A. Shea and G. K. Ackers, "The OR control system of bacteriophage lambda. A physical-chemical model for gene regulation," Journal of Molecular Biology, vol. 181, no. 2, pp. 211230, 1985.
[3] J. Reinitz and J. R. Vaisnys, "Theoretical and experimental analysis of the phage lambda genetic Switch implies missing levels of co-operativity," Journal of Theoretical Biology, vol. 145, no. 3, pp. 295-318, 1990.

[4] M. Santillán and M. C. Mackey, "Why the lysogenic state of phage $\lambda$ is so stable: a mathematical modeling approach," Biophysical Journal, vol. 86, no. 1 I, pp. 75-84, 2004.

[5] S. Wang, Y. Zhang, and Q. Ouyang, "Stochastic model of coliphage lambda regulatory network," Physical Review E, vol. 73, no. 4, Article ID 041922, 2006.

[6] R. Zhu, A. S. Ribeiro, D. Salahub, and S. A. Kauffman, "Studying genetic regulatory networks at the molecular level: delayed reaction stochastic models," Journal of Theoretical Biology, vol. 246, no. 4, pp. 725-745, 2007.

[7] D. Laschov and M. Margaliot, "Mathematical modeling of the lambda switch: a fuzzy logic approach," Journal of Theoretical Biology, vol. 260, no. 4, pp. 475-489, 2009.

[8] L. Zeng, S. O. Skinner, C. Zong, J. Sippy, M. Feiss, and I. Golding, "Decision making at a subcellular level determines the outcome of bacteriophage infection," Cell, vol. 141, no. 4, pp. 682-691, 2010.

[9] M. Chaves and J. Gouzé, "Exact control of genetic networks in a qualitative framework: the bistable switch example," Automatica, vol. 47, no. 6, pp. 1105-1112, 2011.

[10] E. Aurell and K. Sneppen, "Epigenetics as a first exit problem," Physical Review Letters, vol. 88, no. 4, Article ID 048101, 4 pages, 2002.

[11] X.-M. Zhu, L. Yin, L. Hood, and P. Ao, "Robustness, stability and efficiency of phage $\lambda$ genetic switch: dynamical structure analysis," Journal of Bioinformatics and Computational Biology, vol. 2, no. 4, pp. 785-818, 2004.

[12] T. Tian and K. Burrage, "Bistability and switching in the lysis/lysogeny genetic regulatory network of bacteriophage $\lambda$," Journal of Theoretical Biology, vol. 227, no. 2, pp. 229-237, 2004.

[13] C. Lou, X. Yang, X. Liu, B. He, and Q. Ouyang, "A quantitative study of $\lambda$-phage SWITCH and its components," Biophysical Journal, vol. 92, no. 8, pp. 2685-2693, 2007.

[14] L. M. Anderson and H. Yang, "DNA looping can enhance lysogenic CI transcription in phage lambda," Proceedings of the National Academy of Sciences of the United States of America, vol. 105, no. 15, pp. 5827-5832, 2008.

[15] H. Zhu, T. Chen, X. Lei, W. Tian, Y. Cao, and P. Ao, "Understand the noise of CI expression in phage lysogen," in Proceedings of the 31st Chinese Control Conference (CCC '12), pp. 7432-7436, 2012.

[16] A. Bakk and R. Metzler, "In vivo non-specific binding of $\lambda$ CI and Cro repressors is significant," FEBS Letters, vol. 563, no. 1-3, pp. 66-68, 2004.

[17] A. Bakk and R. Metzler, "Nonspecific binding of the OR repressors CI and Cro of bacteriophage $\lambda$," Journal of Theoretical Biology, vol. 231, no. 4, pp. 525-533, 2004.

[18] T. S. Gardner, C. R. Cantor, and J. J. Collins, "Construction of a genetic toggle switch in Escherichia coli," Nature, vol. 403, no. 6767, pp. 339-342, 2000.

[19] D. S. Burz and G. K. Ackers, "Cooperativity mutants of bacteriophage $\lambda$ cI repressor: temperature dependence of self-assembly," Biochemistry, vol. 35, no. 10, pp. 3341-3350, 1996.

[20] W. S. Hlavecek and M. A. Savageau, "Subunit structure of regulator proteins influences the design of gene circuitry: analysis of perfectly coupled and completely uncoupled circuits," Journal of Molecular Biology, vol. 248, no. 4, pp. 739-755, 1995. 
[21] E. Aurell, S. Brown, J. Johanson, and K. Sneppen, "Stability puzzles in phage $\lambda$," Physical Review E, vol. 65 , no. 5, Article ID 051914, 9 pages, 2002.

[22] J. W. Little, D. P. Shepley, and D. W. Wert, "Robustness of a gene regulatory circuit," The EMBO Journal, vol. 18, no. 15, pp. 42994307, 1999.

[23] A. Bakk, R. Metzler, and K. Sneppen, "Sensitivity of OR in phage $\lambda$," Biophysical Journal, vol. 86, no. 1 I, pp. 58-66, 2004.

[24] T. Gedeon, K. Mischaikow, K. Patterson, and E. Traldi, "Binding cooperativity in phage $\lambda$ is not sufficient to produce an effective switch," Biophysical Journal, vol. 94, no. 9, pp. 3384-3392, 2008.

[25] M. J. Morelli, P. R. Wolde, and R. J. Allen, "DNA looping provides stability and robustness to the bacteriophage $\lambda$ switch," Proceedings of the National Academy of Sciences of the United States of America, vol. 106, no. 20, pp. 8101-8106, 2009.

[26] M. Ptashne, A Genetic Switch: Phage Lambda and Higher Organisms, Cell and Blackwell Scientific, Cambridge, Mass, USA, 1992.

[27] C. Zong, L. So, L. A. Sepúlveda, S. O. Skinner, and I. Golding, "Lysogen stability is determined by the frequency of activity bursts from the fate-determining gene," Molecular Systems Biology, vol. 6, no. 1, article 440, 2010.

[28] R. T. Sauer, M. J. Ross, and M. Ptashne, "Cleavage of the lambda and P22 repressors by recA protein," The Journal of Biological Chemistry, vol. 257, no. 8, pp. 4458-4462, 1982.

[29] M. Ptashne, A Genetic Switch: Phage Lambda Revisited, Cold Spring Harbor Laboratory Press, 2004.

[30] J. W. Roberts and C. W. Roberts, "Proteolytic cleavage of bacteriophage lambda repressor in induction," Proceedings of the National Academy of Sciences of the United States of America, vol. 72, no. 1, pp. 147-151, 1975.

[31] N. Chia, I. Golding, and N. Goldenfeld, " $\lambda$-prophage induction modeled as a cooperative failure mode of lytic repression," Physical Review E, vol. 80, no. 3, Article ID 030901, 2009.

[32] P. J. Darling, J. M. Holt, and G. K. Ackers, "Coupled energetics of $\lambda$ cro repressor self-assembly and site-specific DNA operator binding II: cooperative interactions of cro dimers," Journal of Molecular Biology, vol. 302, no. 3, pp. 625-638, 2000.

[33] K. Brooks and A. J. Clark, "Behavior of lambda bacteriophage in a recombination deficienct strain of Escherichia coli," Journal of Virology, vol. 1, no. 2, pp. 283-293, 1967.

[34] J. L. Cherry and F. R. Adler, "How to make a biological switch," Journal of Theoretical Biology, vol. 203, no. 2, pp. 117-133, 2000.

[35] A. Arkin, J. Ross, and H. H. McAdams, "Stochastic kinetic analysis of developmental pathway bifurcation in phage $\lambda$ infected Escherichia coli cells," Genetics, vol. 149, no. 4, pp. 16331648, 1998.

[36] P. S. Swain, M. B. Elowitz, and E. D. Siggia, "Intrinsic and extrinsic contributions to stochasticity in gene expression," Proceedings of the National Academy of Sciences of the United States of America, vol. 99, no. 20, pp. 12795-12800, 2002.

[37] G. Nilsson, J. G. Belasco, S. N. Cohen, and A. von Gabain, "Growth-rate dependent regulation of mRNA stability in Escherichia coli," Nature, vol. 312, no. 5989, pp. 75-77, 1984.

[38] S. Sastry, Nonlinear Systems: Analysis, Stability and Control, Interdisciplinary Applied Mathematics: Systems and Control, Springer, 1999.

[39] X.-M. Zhu, L. Yin, L. Hood, and P. Ao, "Calculating biological behaviors of epigenetic states in the phage $\gamma$ life cycle," Functional and Integrative Genomics, vol. 4, no. 3, pp. 188-195, 2004.
[40] I. B. Dodd, A. J. Perkins, D. Tsemitsidis, and J. B. Egan, "Octamerization of $\lambda$ CI repressor is needed for effective repression of PRM and efficient switching from lysogeny," Genes and Development, vol. 15, no. 22, pp. 3013-3022, 2001. 

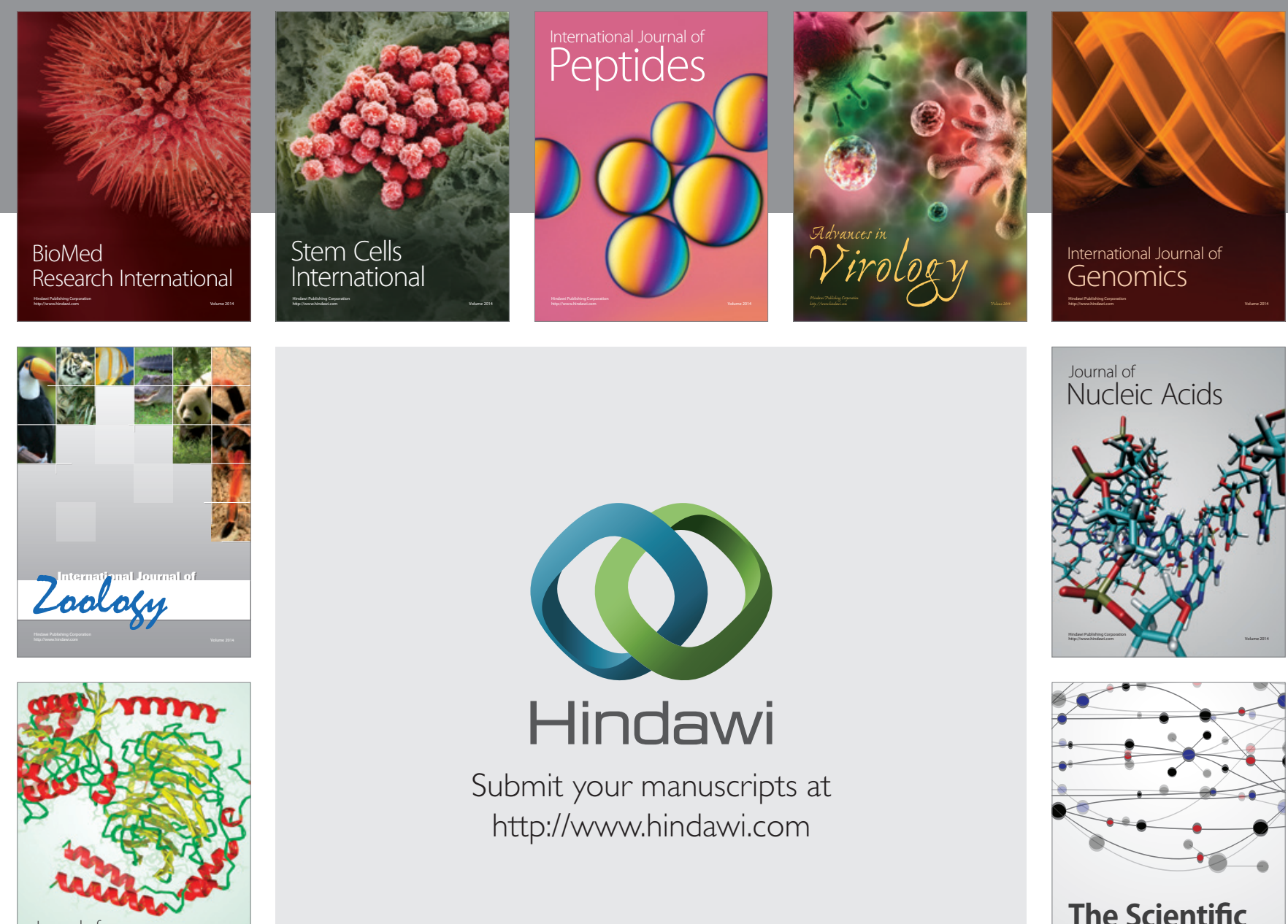

Submit your manuscripts at

http://www.hindawi.com

Journal of
Signal Transduction
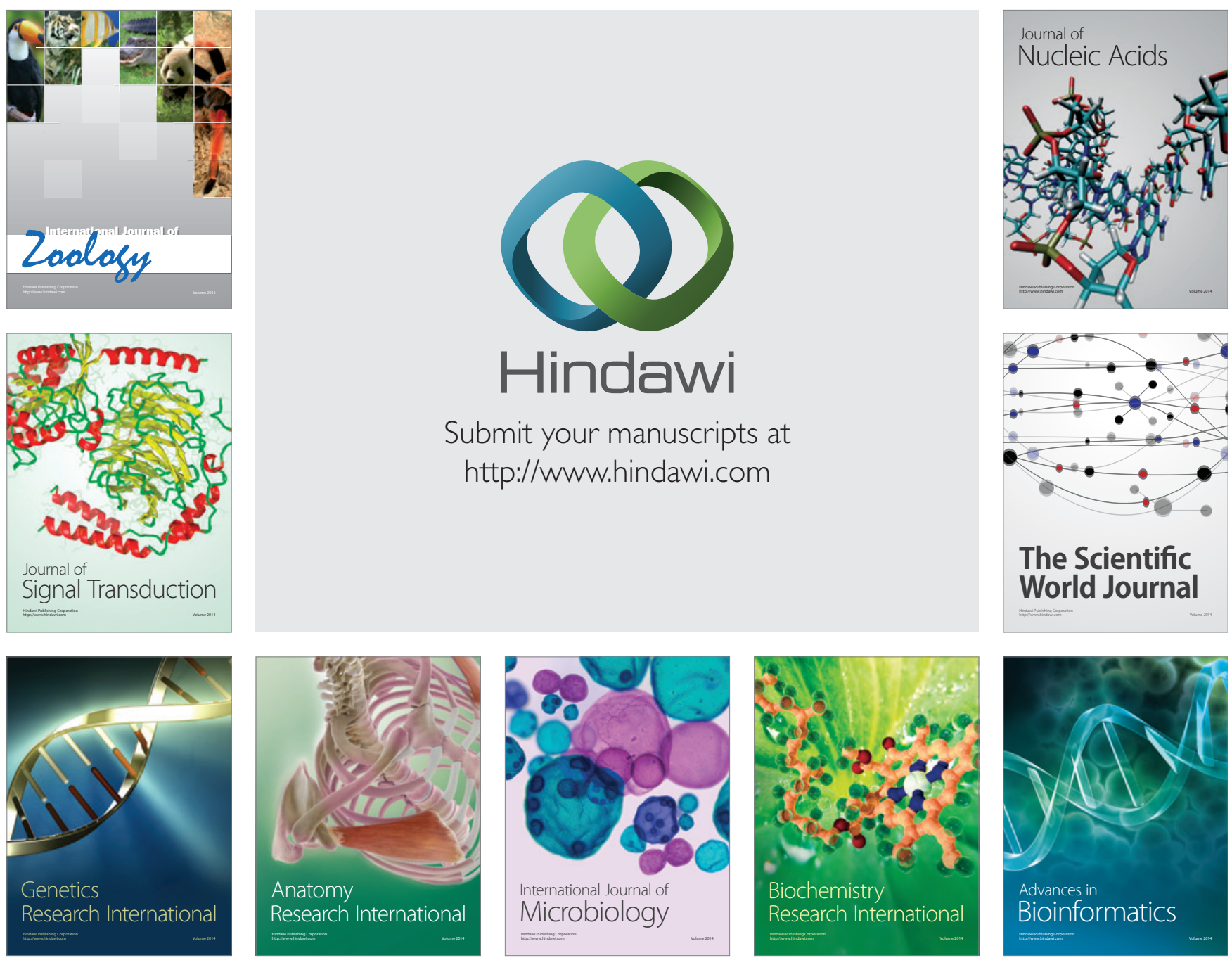

The Scientific World Journal
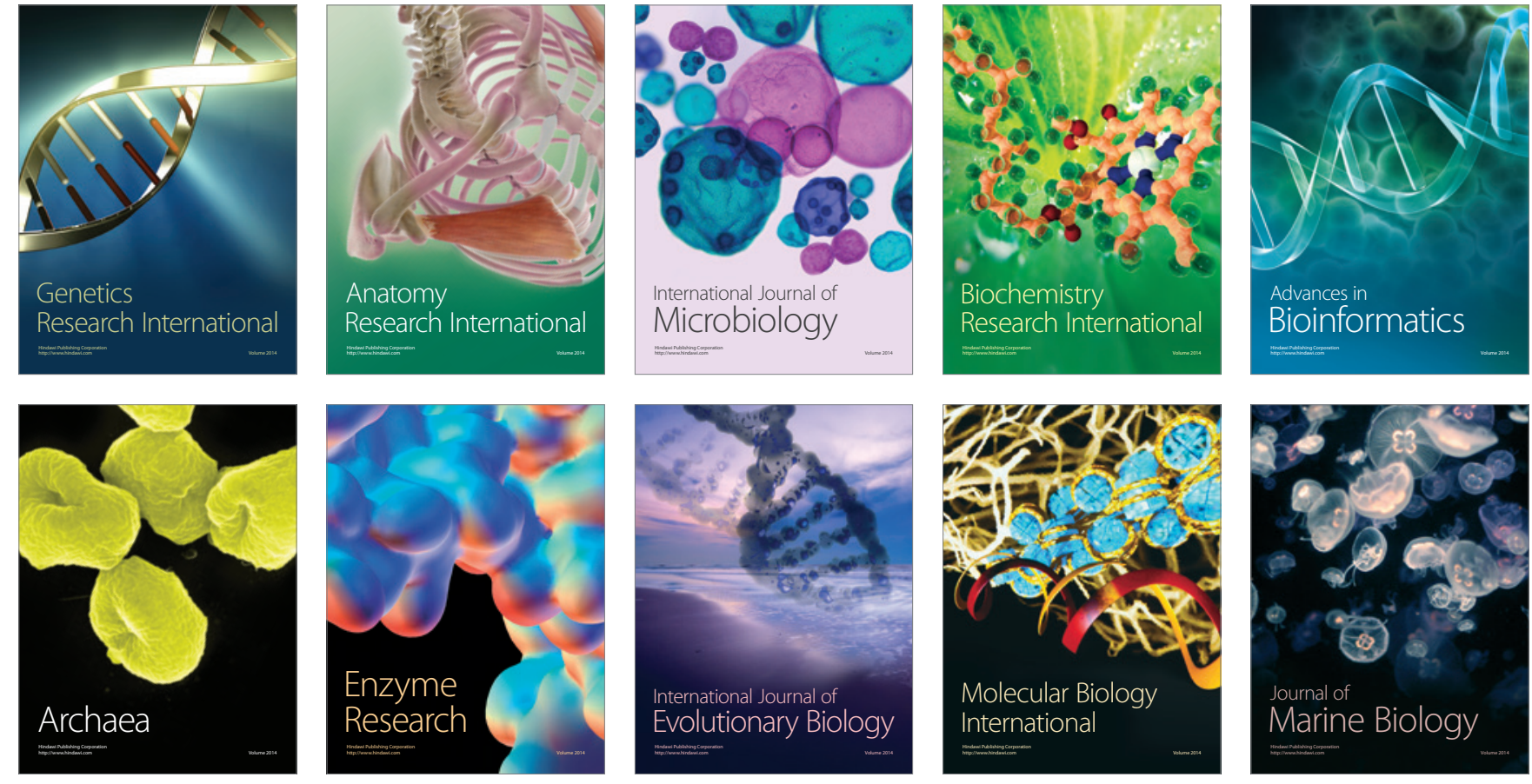